\section{P111 DEVELOPMENTAL PHARMACOGENETICS OF CYP2D6 IN CHINESE CHILDREN: LORATADINE AS A PROBE DRUG}

${ }^{1} \mathrm{Q}$ Li, ${ }^{1}$ Wu Y-E, ${ }^{1} \mathrm{~K}$ Wang, ${ }^{1} \mathrm{H}-\mathrm{Y}$ Shi, ${ }^{1} \mathrm{Y}$ Zhou, ${ }^{1} \mathrm{M}$ Kan, ${ }^{1} \mathrm{Y}$ Zheng, ${ }^{1} \mathrm{G}-\mathrm{X}$ Hao, ${ }^{1} \mathrm{X}-\mathrm{M}$ Yang, ${ }^{1} Y$-L Yang, ' ${ }^{1}$ Su L-Q, ${ }^{2} X-L$ Wang, ${ }^{3} E$ Jacqz-Aigrain, ${ }^{1} W$ Zhao*. 'Shandong University, Jinan; ${ }^{2}$ Beijing Children's Hospital, Beijing, China; ${ }^{3}$ Hôpital Robert Debré, Paris, France

10.1136/archdischild-2019-esdppp.149

Background Although the understanding of CYP2D6 developmental pharmacogenetics in children has made great progress, the current findings are mainly focused on Caucasian children. Given the clear ethnicity difference of CYP2D6 pharmacogenetic profile, there are still unmet needs in understanding developmental pharmacogenetics in inter-ethnic population. We sought to use loratadine as a probe drug to evaluate the effects of ontogeny and pharmacogenetics on the developmental pattern of CYP2D6 in Chinese paediatric patients.

Methods Chinese children receiving loratadine treatment were enrolled in the present study. The metabolic ratio (MR) of loratadine converted to desloratadine [desloratadine concentrations/loratadine concentrations] of trough concentrations samples at steady-state condition was used as a surrogate of CYP2D6 activity. Loratadine and desloratadine were determined by LC/MS/MS method and variants of CYP2D6 were genotyped.

Results A total of 40 patients were available for final analysis. The mean age was 4.6 (range 0.5-9.0) years and the mean weight was 19.9 (range 9.0- 42.0) kg. The MR was significantly higher in homozygous wild-type subjects compared with CYP2D6*10 subjects $(16.94 \pm 14.12$ versus 9.37 $\pm 7.54, \mathrm{p}=$ 0.028 ). Weight was also found to be significantly corrected with MR ( $\mathrm{p}=0.030)$.

Conclusions The developmental pharmacogenetics of CYP2D6 in Chinese children was evaluated using loratadine as a probe drug. Weight and CYP2D6 genotype showed independently significant impacts on MR.

Disclosure(s) Nothing to disclose.

\section{P112 PREDICTION OF FREE CEFTRIAXONE CONCENTRATION IN CHILDREN: DISEASE AND MATURATION DO MATTER}

${ }^{1} \mathrm{H}-\mathrm{Y}$ Shi, ${ }^{1} \mathrm{X}$ Huang, ${ }^{1} \mathrm{Q} \mathrm{Li},{ }^{1} \mathrm{~W}$ U Y-E, ${ }^{1} \mathrm{MW}$ Khan, ${ }^{1} \mathrm{Y}$ Zhou, ${ }^{1} \mathrm{Y}$ Zheng, ${ }^{1} \mathrm{G}-\mathrm{X}$ Hao, ${ }^{1} \mathrm{X}-$ $\mathrm{M}$ Yang, ${ }^{1} \mathrm{Y}$-L Yang, ${ }^{2} \mathrm{E}$ Jacqz-Aigrain, ${ }^{3} \mathrm{Y}-\mathrm{M}$ Cui, ${ }^{1} \mathrm{~W}$ Zhao*. ${ }^{1}$ Shandong University, Jinan, China; ${ }^{2}$ Hôpital Robert Debré, Paris, France; ${ }^{3}$ Peking University First Hospital, Bejing, China

\subsection{6/archdischild-2019-esdppp.150}

Background To evaluate the predictive ability of the existing formula to measure free ceftriaxone levels in children, and optimize the formula by adding disease and maturation factors.

Methods Fifty children receiving ceftriaxone were evaluated, and the predictive performance of the different equations were assessed by mean absolute error (MAE), mean prediction error (MPE) and linear regression of predicted vs. actual free levels.

Results The average free ceftriaxone concentration was $2.11 \pm$ $9.51 \mu \mathrm{g} / \mathrm{ml}$. The predicted free concentration was $1.15 \pm$ $4.39 \mu \mathrm{g} / \mathrm{ml}$ with the in vivo binding equation, which increased to $1.58 \pm 7.73 \mu \mathrm{g} / \mathrm{ml}$ and $2.01 \pm 9.53 \mu \mathrm{g} / \mathrm{ml}$ when adjusted for age (disease adapted equation), and age and albumin (disease-maturation equation) respectively. The average MAE values were 0.48 (in vivo banding equation), 0.34 (disease adapted equation) and 0.41 (disease maturation equation). The average MPE values were -0.41 (in vivo binding equation), 0.14 (disease adapted equation) and 0.09 (disease maturation equation). The respective linear regression equations and coefficients were $y=1.8647 \mathrm{x}+1.0731(\mathrm{R} 2=0.7398), \quad \mathrm{y}=1.1455 \mathrm{x}$ $+0.8414(\mathrm{R} 2=0.8674)$, and $\mathrm{y}=0.9664 \mathrm{x}(\mathrm{R} 2=0.8641)$ for the in vivo binding, disease adapted and disease maturation equations respectively.

Conclusion Compared to the in vivo binding equation, the disease adapted and disease maturation equations showed lower MAE and MPE values, and the latter showed the lowest MPE value. In addition, the slope of the disease maturation equation was closer to 1 compared to the other two. Therefore, the optimized disease maturation equation should be used to measure free ceftriaxone levels in children.

Disclosure(s) Nothing to disclose.

\section{P113 POPULATION PHARMACOKINETICS AND DOSING OPTIMIZATION OF AZLOCILLIN IN NEONATES}

W Zhao*, TINN-Global Study Group. Shandong University, Jinan, China

\subsection{6/archdischild-2019-esdppp.151}

Background Azlocillin is prescribed for the treatment of infections in neonatal clinical practice, however, the optimized dose is still questionable due to the lack of pharmacokinetic study Thus, we aim to evaluate the population pharmacokinetics of azlocillin and optimize dosing regimen in order to improve azlocillin treatment in neonates.

Methods This is a prospective, open label pharmacokinetic study of azlocillin. Blood samples were collected using an opportunistic sampling design. Theplasma concentrations ofazlocillinwere determined by high performance liquid chromatography method with UV detection. Population pharmacokinetic-pharmacodynamic analysis was performed using NONMEM software.

Results Ninety-five neonates (postmenstrual age (PMA) range 32.1-42.0 weeks) were included in this study. A total of 167 azlocillin concentrations were available for the final analysis. A one-compartment model with first-order elimination best fitted the data. Covariate analysis demonstrated that current weight, birth weight and postnatal age had significant effect on azlocillin pharmacokinetics. MonteCarlo simulation demonstrated that for the common pathogens with MIC of $8 \mathrm{mg} /$ liter, the currently used dosage regimen $(100 \mathrm{mg} / \mathrm{kg}, \mathrm{q} 12 \mathrm{~h})$ resulted in $61.2 \%$ of newborns achieved the pharmacodynamic target (drug concentrations above MIC during 70\% of the dosing interval) with a potential risk of underdosing. When shortening the dosing interval to 8 hours, the target could be achieved in $89.3 \%$ of patients, using the MIC break point of $8 \mathrm{mg} /$ liter.

Conclusion The population pharmacokinetics characteristics of azlocillin were assessed in neonates. An optimal dosage regimen of azlocillin was established based on developmental pharmacokinetics-pharmacodynamics in this vulnerable population. 
Disclosure(s) Nothing to disclose.

\section{P114 POPULATION PHARMACOKINETICS AND DOSING OPTIMIZATION OF CEFEPIME IN NEONATES}

W Zhao*, TINN-Global Study Group. Shandong University, Jinan, China

\subsection{6/archdischild-2019-esdppp.152}

Objective Cefepime, a fourth-generation cephalosporin, is used in the treatment of severe nosocomial infections in neonates. Pharmacokinetics of cefepime was limited. Therefore, we aimed to study the population pharmacokinetics of cefepime and optimize cefepime regimen in preterm and term neonates. Methods Blood samples were obtained from neonates treated with cefepime using an opportunistic sampling design. Concentration of cefepime was determined by high performance liquid chromatography. Population pharmacokinetics analysis was conducted using NONMEM software.

Results Sparse pharmacokinetic samples $(n=100)$ from 85 neonatal patients were available for analysis. A one-compartment model with first-order elimination was used to describe the pharmacokinetics of cefepime. Covariate analysis showed that current weight, postmenstrual age and serum creatinine concentration had tremendous influence on pharmacokinetics of cefepime. Monte Carlo simulation indicated that current dosage regimen $(30 \mathrm{mg} / \mathrm{kg}, \mathrm{q} 12 \mathrm{~h}$ ) was correlated with high risk of underdosing in neonates. To achieve the target rate of $70 \%$ of patients get free drug concentration above MIC during $70 \%$ of dosing interval, $30 \mathrm{mg} / \mathrm{kg} \mathrm{q} 8 \mathrm{~h}$ was required for all neonates, using susceptibility breakpoint of $4 \mathrm{mg} / \mathrm{L}$.

Conclusion The population pharmacokinetics characteristics of cefepime were evaluated in neonates. Based on simulation, different dosage regimens were required depending on the postmenstrual age and pathogens.

Disclosure(s) Nothing to disclose.

\section{P115 EFFECTS OF THE CLINICAL PHARMACIST'S INTERVENTION ON RATIONALITY OF PARENTERAL NUTRITION}

M Zhu*, J Xu. Nanjing Children's Hospital, Nanjing, China

10.1136/archdischild-2019-esdppp.153

Objective Through STRONGkids ${ }^{1}{ }^{2}$ (screening tool risk on nutritional status and growth of children) to observe the influences on nutritional indicators and postoperative recovery of different nutritional risk levels of children with intussusception in the use of parenteral nutrition support. Through educating and interventing the doctors to promote the use of parenteral nutrition more reasonable and the hospitalization costs lower. ${ }^{3}$ Methods Children were grouped according to different scores of STRONGkids, ${ }^{4} 5$ and each group was divided into two groups $\mathrm{A}$ and $\mathrm{B}$ according to using parenteral nutrition only or no nutrition support at all. The proportion of the two groups, nutritional indicators and postoperative recovery of the children after surgery were compared to observe the parenteral nutrition usage rate of different groups, and the use of parenteral nutrition was necessary or not. The clinical pharmacist intervened the doctors according to the research results. 1 year later, the indicators above were compared again.
Results There were no significant differences on nutritional indicators and postoperative recovery in 1-2 score groups between group $\mathrm{A}$ and $\mathrm{B}$, but the hospitalization cost in group A was significantly higher than that in group B. In 3-score group of children, the decreases of weight, prealbumin and retinol binding protein were more significant in group $B$ than in group A, and the hospitalization days of group A were significantly shorter than group B. The incidence of adverse reactions of using parenteral nutrition was significantly higher. According to above results, the clinical pharmacist instructed doctors to improve the indication of parenteral nutrition according to the relevant guidelines. 1 year later, the usage rate of parenteral nutrition dropped in 1-2 score groups. The incidence of adverse reactions and the costs of hospitalization were significantly decreased.

Conclusions The clinical pharmacist played an important role in promoting the rational use of parenteral nutrition. ${ }^{6} 7$

\section{REFERENCES}

1. Teixeira AF, Viana KD.Nutritional screening in hospitalized pediatric patients: a systematic review.[J]J Pediatr (Rio J) 2016, 92(4):343-352.

2. Forga L, Bolado F, Goñi MJ,et al. Low serum levels of prealbumin, retinol binding protein, and retinol are frequent in adult type 1 diabetic patients.J Diabetes Res 2016;2016:2532108. doi: 10.1155/2016/2532108. Epub 2016 Nov 29.

3. Pediatric Collaborative Group, Society of Parenteral and Enteral Nutrition. Guidelines for pediatric clinical application of enteral and parenteral nutritional support in China[J]. Zhonghua Er Ke Za Zhi, 2010, 48(6):436-441.

4. Abunnaja S, Cuviello A, Sanchez JA.Enteralandparenteral nutritionin the perioperative period: state of the art[J].Nutrients. 2013, 5(2):608-623.

5. Yi F, Ge L, Zhao J, Lei Y,et al.Meta-analysis:total parenteral nutritionversustotalenteral nutritionin predicted severe acute pancreatitis[J].Intern Med. 2012, 51 (6):523-530.

Disclosure(s) Nothing to disclose.

\section{\begin{tabular}{l|l} 
P116 PROPHYLACTIC USE OF ENOXAPARIN DURING \\
\hline
\end{tabular} BARIATRIC SURGERY IN ADOLESCENTS WITH SEVERE OBESITY}

$1,2^{2}$ Z Ziesenitz ${ }^{*},{ }^{3}$ J Vaughns, ${ }^{4}$ E Williams, ${ }^{5} \mathrm{E}$ Nadler, ${ }^{6} \mathrm{G}$ Mikus, ${ }^{1,4} \mathrm{~J}$ van den Anker. ${ }^{1}$ Pediatric Pharmacology and Pharmacometrics, University Children's Hospital Basel (UKBB), Basel, Switzerland; ' 2 Pediatric Cardiology, University Children's Hospital Heidelberg, Heidelberg, Germany; ${ }^{3}$ Anesthesia and Pain Medicine and Clinical Pharmacology; ${ }^{4}$ Clinical Pharmacology; ${ }^{5}$ Surgery, Children's National Health System, Washington, DC, USA; ${ }^{6} \mathrm{Clinical}$ Pharmacology and Pharmacoepidemiology, Universty Hospital Heidelberg, Heidelberg, Germany

\subsection{6/archdischild-2019-esdppp.154}

Background Severe obesity predisposes adults and youth to a higher risk of venous thromboembolism (VTE). Enoxaparin is frequently used for their VTE management. This study evaluates a BMI-stratified prophylactic dosing regimen of enoxaparin in severely obese adolescents undergoing bariatric surgery.

Methods This prospective study enrolled severely obese adolescents aged 12-20 years undergoing laparoscopic sleeve gastrectomy. Prophylactic enoxaparin was dosed at $40 \mathrm{mg}$ SC (for a BMI less than $50 \mathrm{~kg} / \mathrm{m}^{2}$ ) and $60 \mathrm{mg}$ SC (for a BMI equal to or greater than or $50 \mathrm{~kg} / \mathrm{m}^{2}$ ). Blood samples were drawn until 12 hrs post-dose. Plasma Anti-Factor Xa (Anti-FXa) activity was used as a surrogate marker for enoxaparin plasma concentration and pharmacokinetics were assessed using non-compartmental PK analysis. The primary efficacy outcome was the anti-FXa activity 4-6 hours after dosing, and the primary endpoint was the proportion of patients who reached prophylactic anti-FXa activity of $0.1-0.3 \mathrm{U} / \mathrm{mL}$ between $4-6$ hours after dosing. 